\title{
A Possible Use of Fiber-Optic Ring Resonator as a Nano-Scale Optical Sensor for Detection of Physical Quantities
}

\author{
Melika E. Se raji ${ }^{1}$, Faramarz E. Se raji ${ }^{2, *}$, H. Golnabi ${ }^{1,3}$ \\ ${ }^{1}$ Department of Phy sics, Science and Research Branch, Islamic Azad Univ., Tehran, Iran \\ ${ }^{2}$ Optical Communication Group, Iran Telecom Research Center, Tehran, Iran \\ ${ }^{3}$ Institute of Water and Energy, Sharif University of Technology, Tehran, Iran
}

\begin{abstract}
This paper presents a proposed fiber-optic ring resonator with a resonance loop made of a conventional single-mode optical fiber as a nano-scale sensor for measurement of physical quantities, such as pressure and temperature. The operational theory of the resonator as an optical sensor is presented, where the effects of characteristic parameters of the resonator on the sensor response is investigated. It is shown that the behavior of sensor response with respect to some particular physical quantities is linear and is capable of detecting measurand variations of the order of 100 nanometers. The proposed sensor is suitable for design of hydrophones used in submarine co mmunications.
\end{abstract}

Keywo rds Nano-scale, Optical Sensors, Finer-optic Ring Resonator

\section{Introduction}

In the last two decades, fiber-optic ring resonators (FORRs) with fiber and waveguide structures have been used in several applications such as biosensors[1], optical switching[2], add/drop multip lexers [3], laser resonators [4], dispersion compensators[5], optical bistability[6], optical filters[7]. Recently, performance analyses of FORR under steady and dynamic states are reported, where the resonator responses in terms of characteristic parameters, are investigated $[8,9]$. More recently, the use of FORR for tunable optical filters is analysed, where the resonance loop of the FORR was made of photonic crystal fibers[10].

A nano-opto-mechanical pressure sensor based on ring resonator is designed, fabricated and characterized for pressures ranging from $0 \mathrm{kPa}$ to $60 \mathrm{kPa}$. The sensitivity and resolution of the pressure sens or is $1.47 \mathrm{p} \mathrm{m} / \mathrm{kPa}$ and $1.36 \mathrm{kPa}$, respectively[11]. A novel refractometric sensor in the form of an embedded optical nanowire loop resonator is investigated experimentally, where a sensitivity as high as $700 \mathrm{~nm} / \mathrm{RIU}$ and a refractive index resolution as low as $10^{-7}$ is achieved[12].

A digital optical sensor based on two cascaded ring resonators with different free spectral ranges to measure refractive index is proposed, which has shown a high

* Corresponding author:

feseraji@itrc.ac.ir(Faramarz E. Seraji)

Published online at http://journal.sapub.org/optics

Copyright (C) 2012 Scientific \& Academic Publishing. All Rights Reserved sensitivity of the order of $105 \mathrm{~nm} / \mathrm{RIU}$ [13].

Yet in another report, a method is proposed theoretically to simultaneously measure refractive-index (RI) and temperature changes using optical ring resonators. The analysis has shown that the RI and temperature detection limits can be achieved on the order of $10^{-7} \mathrm{RI}$ unit and $10^{-3} \mathrm{~K}$ at a wavelength of $780 \mathrm{~nm}$ [14].

Unlike the other methods[11-13], in the present paper, by considering the steady state response of the FORR[9], an analysis of possible use of an FORR as a nano-scale sensor for measurement of physical quantities such as pressure and temperature is presented. In the present analysis, it is shown that if the resonance loop is exposed to a physical quantity of mechanical nature, the output characteristics of the FORR will change linearly in a specified range of wavelengths in response to nano-scale variations of sensing length.

\section{Structure of FORR Based Sensor}

A basic structure of an FORR is made of a $2 \times 2$ optical directional coupler with a resonance loop constructed by connecting an input port to one of the output port. In Fig. 1, the proposed FORR to measure a physical quantity is shown, where its resonance loop of length $L$ is exposed to a physical quantity, such as acoustic waves. The characteristic parameters of the FORR are loop transmission coefficient $\alpha$, coupler coupling coefficient $\kappa$, coupler insertion loss $\gamma$, and loop delay time $\tau$.

For our analysis of the FORR response as an optical sensor, without of loss of generality, the attenuation of the loop is 
assumed to be only due to macrobending, while other attenuation mechanisms such as intrinsic and joint losses are neglected. The loop transmission coefficient is obtained by $\alpha=\exp \left(-2 \alpha_{0} L\right)$, where $\alpha_{0}$ is the attenuation coefficient due to macrobening loss in terms of $\mathrm{dB} / \mathrm{m}$.

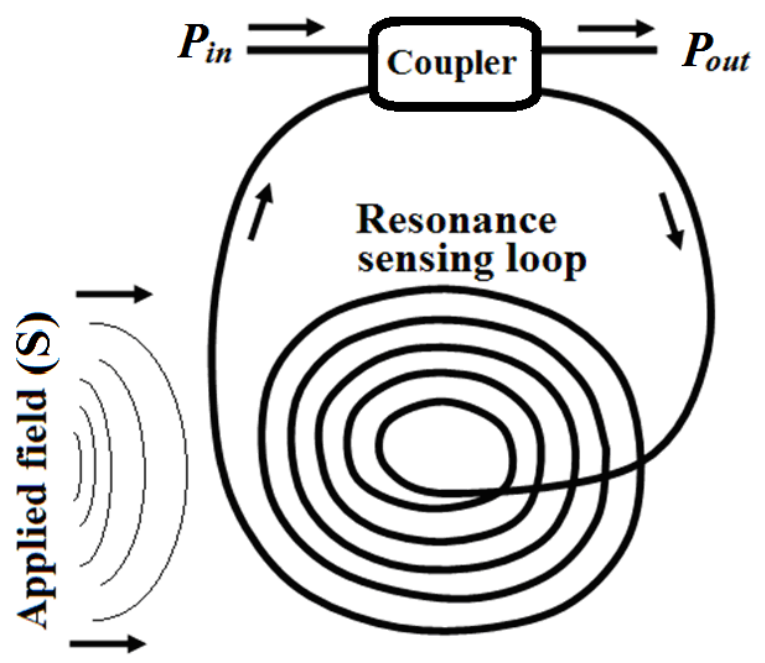

Figure 1. The proposed nano-scale sensor based on FORR

When the resonance loop of the FORR is exposed to the physical quantity, the phase of the propagating mode in the loop suffers some changes. If $L$ is the average length of the loop and $n$ is the refractive index of the fiber core, the phase change $(\delta \phi / \delta S)$ in the optical path length of $n L$, due to an applied physical quantity $S$, will be as $\delta \phi / \delta S=(2 \pi / \lambda)[L \delta n / \delta S+n \delta L / \delta S][11]$, which shows that the physical quantity causes independent changes of the loop length and the refractive index of the core.

If we use a birefringent fiber for the resonance loop, the polarization phase angle obtained in propagating mode due to two perpendicular polarization modes $\left(n_{p}, n_{q}\right)$ is given as $\phi_{p q}=(2 \pi / \lambda)\left(n_{p}-n_{q}\right) L[11]$. On the other hand, the average phase of propagating mode with angular frequency of $\omega$ in the loop with delay time of $\tau$ will be $\omega \tau$. Therefore, from polarization phase angle $\phi_{p q}$, the loop delay time is obtained as $\tau=\left(n_{p}-n_{q}\right) L / c$, where $c$ is the speed of light in a vacuum. If $P_{\text {in }}(t)$ is the signal of angular frequency $\omega$ from a laser diode (LD) modulated by $\omega_{\mathrm{m}}$, is applied to the input port of the sensor, the response after $N$ circulations of the propagating mode in the loop, can be expressed as[10]:

$$
\begin{aligned}
P_{\text {out }}^{(N)} & =-j A \sqrt{1+k_{\mathrm{m}} \sin \left(\omega_{\mathrm{m}} t\right)} \exp \left[-j \beta \cos \left(\omega_{\mathrm{m}} t+\varphi_{\mathrm{FM}}\right)\right]+ \\
j B & \sum_{N=1}^{N=\infty}\left\{\begin{array}{l}
C^{(N-1)} \sqrt{1+k_{\mathrm{m}} \sin \left[\omega_{\mathrm{m}}(t-N \tau)\right]} \times \\
\exp \left\{j\left[-\beta \cos \left[\omega_{\mathrm{m}}(t-N \tau)+\varphi_{\mathrm{FM}}\right]-N(\omega \tau+\pi / 2)\right]\right\}
\end{array}\right\}
\end{aligned}
$$

where $\quad A=[\kappa(1-\gamma)]^{1 / 2}, \quad B=(1-\kappa)(1-\gamma) \alpha^{1 / 2}, \quad$ and $C=[\alpha \kappa(1-\gamma)]^{1 / 2}$ are constants for a given FORR, $\beta=k_{f} I_{\mathrm{m}} / f_{m}$ is the modulation coefficient of the LD signal, $k_{f}$ is the optical frequency deviation $(\mathrm{Hz} / \mathrm{mA}), f_{m}$ is the modulation frequency, $I_{\mathrm{m}}$ is the peak of modulating current of LD, $k_{m}$ is the amplitude modulation coefficient, and $\varphi_{F M}$ is the angle between optical frequency deviation and amp litude modulation current of the LD.

\section{Response of the Sensor}

The steady state response of the FORR in terms of phase $\omega \tau$ of circulating field through the resonance loop in the wavelength range of 1.4325 to $1.4410 \mu \mathrm{m}$ from Eq. (4) is illustrated in Fig. 2, using different values of loop length $L$ and the following specific parameters:

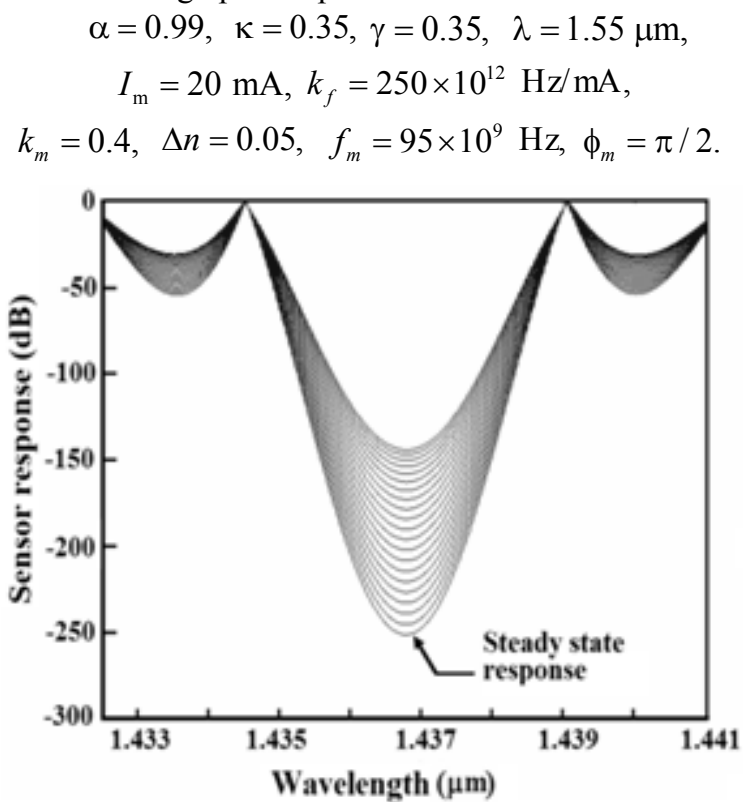

Figure 2. Sensor responses under steady state and applied fields as a function wavelength

Referring to Figure 1, a measurand physical quantity, applied all along the resonance loop, would cause a variation in the length, affects the response of the FORR as a sensor, as shown in Figure 2. In this figure, the lowest curve indicates the response under the steady state condition at wavelength $1.4367 \mu \mathrm{m}$ and loop length of $L=19.0033 \mathrm{~mm}$, that is when the physical quantity is not yet applied. Other curves at upper levels show the response by changing the loop length due to applied measurand quantity. In Figure 3, the response variation is depicted in terms of loop length variations by a step of $100 \mathrm{~nm}$. In the range of 3.30 to $5.30 \mu \mathrm{m}$ variations of the loop length, the response is quite linear. Over this range, the response variations is from $-251.5 \mathrm{~dB}$ to $-144.6 \mathrm{~dB}$. By more investigation of Figure 2, indicates that the slope of sensor responses $(\Delta \Re / \Delta \lambda)$ incurs decreasing variations due to different applied measurand quantities.

In Figure 3, the slope of the curve $(\Delta \Re / \Delta L)$ can be a characteristic response of a given sensor based on a particular FORR. This curve may be approximated by a linear differential equation of the form exp ressed as: 


$$
\lim _{L \rightarrow 0} \frac{\Delta \mathfrak{R}}{\Delta L}=\frac{d \mathfrak{R}}{d L}=55 \times 10^{3} \Rightarrow \mathfrak{R}=55 \times 10^{3} L+C
$$

where $C=-430$ is a constant of integration, in terms of $\mathrm{dB}$.

The linear nature of Eq. (2) with a sharp slope can provide a sensitive measurement of a measurand physical quantity applied on the resonant sensing loop of the resonator.

By a minute investigation of the response curves in Figure 2 reveals that the sensor response is also dependent on the slope of the individual curve for different loop length variations. In the linear regime of the response curves, a measurand physical quantity is applied and the corresponding slope angles are calculated.

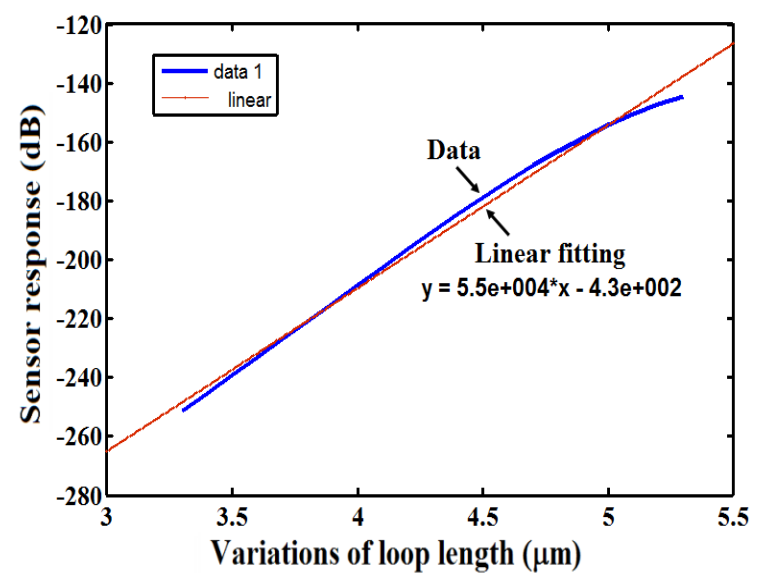

Figure 3. Variation of sensor response in terms of loop length variation at $1.4367 \mu \mathrm{m}$

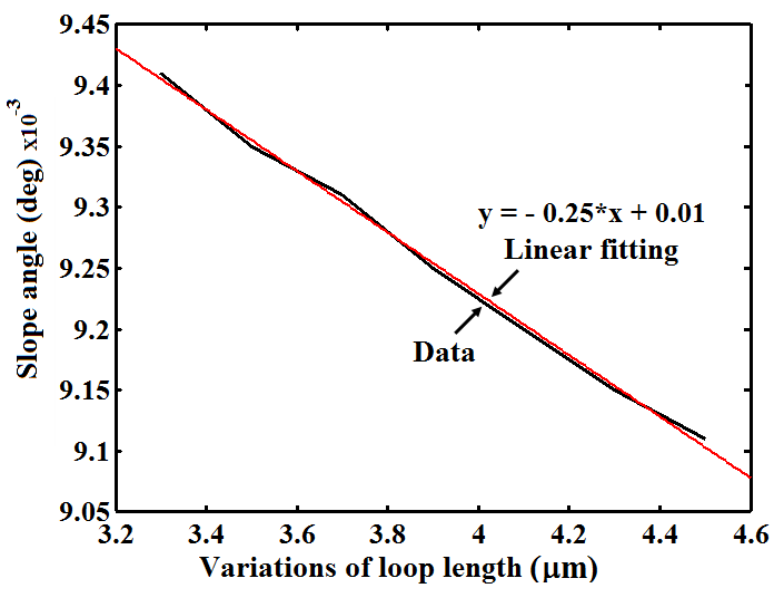

Figure 4. Variations of slope angle versus loop length variations $\Delta L$.

In Figure 4, the variations of slope angle $\theta$ versus loop length $L$ are illustrated for a step of $200 \mathrm{~nm}$. As shown, the range of loop length variations from $3.3 \mu \mathrm{m}$ to $4.5 \mu \mathrm{m}$ has reduced the slope angle from $9.41 \times 10^{-3}$ to $9.11 \times 10^{-3} \mathrm{deg}$.

The slope of the curves $(\Delta \theta / \Delta L)$ in this case can also be approximated by a linear differential equation to represent the response in Figure 4 as follows:

$$
\lim _{L \rightarrow 0} \frac{\Delta \theta}{\Delta L}=\frac{d \theta}{d L}=-0.25 \Rightarrow \theta=-0.25 L+C
$$

where $C$ is a constant of integration equal to 0.01 in terms of degree. In this case, the response curve has a mild slope angle as compared with the case in Eq. (2). indicating a lesser sensitivity to measurand quantity.

\section{Effects of FORR Parameters on Sensor Responses}

The parameters of the FORR and laser source can also influence on the sensor response at some particular conditions $[8,9]$. In our analysis, we focus on effects of the FORR parameters such as $\kappa, \alpha, \gamma$, and for the sake of analytical simplicity, we ignore that of laser source. The effects of other parameters are under investigation whose results will be reported elsewhere.

Using Eq. (1), the effects of parameters $\kappa, \alpha$, and $\gamma$ on the sensor response is illustrated in Figure 5. It is indicated that the effects of $\kappa$ and $\gamma$, lowers the response at two points $\kappa=0.3$ and $\gamma=0.6$ to a minimum level. When the value of $\gamma$ equals to that of $\kappa$, the effects of $\gamma$ will be more pronounced. Moreover, the effects of these parameters on the sensor response in some depicted ranges in Figure 5 behave linearly that may be used as a tool to control the output response.

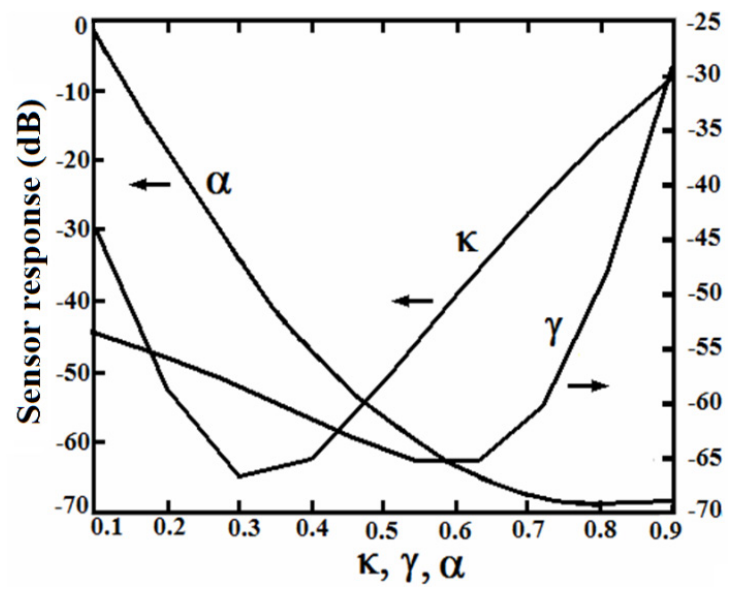

Figure 5. The effects of $\kappa, \alpha$, and $\gamma$ on the sensor response

The effects of transmission coefficient of the resonance loop $\alpha$ on the sensor response are nearly linear in the range of 0.1 to 0.6 . Therefore, the variations of $\alpha$ may also be a means of measurements of physical quantities. As it is shown, over the best linear range of $\alpha$ (from 0.1 to 0.6 ), a detectable variation of $-70 \mathrm{~dB}$ in the sensor response is observed.

\section{Conclusions}

By using a fiber-optic ring resonator, a nano-scale sensor for possible measurements of physical quantities is proposed and its performances are analyzed in terms of characteristic parameters of the ring resonator. The theoretical analys is shows that if a physical quantity, such as under-water acoustic wave, causes a step change of $100 \mathrm{~nm}$ in the resonance loop length, the sensor response would be linear and detectable. In addition, it is shown that by changing the transmission coefficient of the resonance loop via a physical 
quantity in the range of 0.1 to 0.6 , the variations of the sensor response would be linear, which may also be used as a criterion for measurement of physical quantities.

The theoretical results also show that the slope angle of the sensor response changes with applied measurand field. The measurable change may be as low as $100 \mathrm{~nm}$.

It is worthy to mention that the proposed sensor configuration, based on fiber-optic ring resonator, is presented for the first time, which of course requires more theoretical investigations with an experimental data supports. However, the results of this analysis may be used in design of hydrophones utilized in submarine commun ications.

\section{REFERENCES}

[1] Ian M. White, Hesam Oveys, and Xudong Fan, "Integrated multiplexed biosensors based on liquid core optical ring resonators and antiresonant reflecting optical waveguides" Vol. 89, Appl. Phys. Lett., p. 191106-191108, 2006. http://dx.doi.org/10.1063/1.2387112.

[2] Li Junqing, Li Li, Zhao Jiaqun, Li Chunfei, "Ultrafast, low power, and highly stable all-optical switch in MZI with two-arm-sharing nonlinear ring resonator", Opt Commun., Vol. 256, pp. 319-325, 2005.

[3] O. Schwelb, "Crosstalk and Bandwidth of Lossy Micro-ring Add/Drop Multiplexers," Opt. Commun., Vol. 265, No. 1, 2006, pp. 175-179. doi:10.1016/j.optcom.2006.02.055.

[4] S. L. McCall, A.F.J Levi, R. E. Slusher, S. J. Pearton, R. A. Logan. "Whispering-gallery mode microdisk lasers", Appl. Phy. Lett., Vol. 60, p. 289-291, 1992.

[5] Shen Hao, Chen Jian-Ping, Li Xin-Wan, Wang Yi-Ping., "Group Delay and Dispersion Analysis of Compound High Order Microring Resonator All-Pass Filter," Optics Communi- cations, Vol. 262, No. 2, 2006, pp. 200-205. doi:10.1016/j.optcom.2005.12.060.

[6] Y. Dumeige, C. Arnaud and P. Féron, "Combining FDTD with Coupled Mode Theories for Bistability in Micro-Ring Resonators," Optics Communications, Vol. 250, No. 4-6, 2005, pp. 376-383. doi:10.1016/j.optcom.2006.11.065.

[7] Faramarz E. Seraji and F. Asghari, "Tunable optical filter based on Sagnac phase-shift using single optical ring resonator", Opt. \& Laser Technol. Vol. 42, pp. 115-119, 2010, doi:10.1016/j.optlastec.2009.05.008

[8] Faramarz E. Seraji, "Dynamic response of a fiber-optic ring resonator: Analysis with influences of light-source parameters", Prog. Quant. Electron., Vol. 33, No. 2-4. 2009, pp. 110-125. doi:10.1016/j.pquantelec.2009.05.0 01 .

[9] F. E. Seraji, "Steady-State Performance Analysis of FiberOptic Ring Resonator," Progress in Quantum Electronics, Vol. 33, No. 1, 2009, pp. 1-16. doi:10.1016/j.pquantelec.2008.10. 001 .

[10] Kazhal Shalmashi, Faramarz E. Seraji and M. Rezaei Mersagh, "Characteristics of tuneable optical filters using optical ring resonator with PCF resonance loop", Eur. Phys. J. Appl. Phys. (2012) 58: 20502-p 1-p7, doi:10.1051/epjap/2012110364.

[11] X. Zhao, J. M. Tsai, H. Cai, X. M. Ji, J. Zhou, M. H. Bao, Y. P. Huang, D. L. Kwong, and A. Q. Liu, "A nano-opto-mechanical pressure sensor via ring resonator", Vol. 20, No. 8, pp. 8535-8542, Opt. Exp., 2012.

[12] Fei Xu, Valerio Pruneri, Vittoria Finazzi, and Gilberto Brambilla, "An embedded optical nanowire loop resonator refractometric sensor", Vol. 16, No. 2, Opt. Exp., pp. 1062-1067, 2008.

[13] Daoxin Dai, "Highly sensitive digital optical sensor based on cascaded high-Q ring-resonators", Vol. 17, No. 26, Opt. Exp., pp. 23817-23822, 2009.

[14] Nai Lin, Lan Jiang, Sumei Wang, Lei Yuan, and Qianghua Chen, "Simultaneous measurement of refractive index and temperature using a microring resonator", Vol. 10, No. 5, Chin. Opt. Lett., pp. 052802 (1)-(4), 2012.

[15] K.T.V. Gratttan and B.T. Meggitt, Optical fiber sensor technology, Kluwer Academic Publishers, The Netherlands, 2000 . 